\title{
Validação qualitativa de uma escala para medir a carga de trabalho de enfermagem em unidades de internação
}

Qualitative validation of a scale for nursing workloads in hospitalization units Validación cualitativa de una escala para medir la carga de trabajo de enfermería en unidades de internación

\author{
Maria Fuensanta Hellín Gill ${ }^{1}$ io https://orcid.org/0000-0003-0402-0888 \\ Ana Myriam Seva Llor ${ }^{1}$ iD https://orcid.org/0000-0002-0531-9227. \\ Maria Dolores Roldán Valcárcel ${ }^{1}$ iD https://orcid.org/0000-0003-1087-0264 \\ Maria Loreto Maciá Soler ${ }^{2}$ ic https://orcid.org/0000-0002-1801-7607 \\ Marzena Mikla ${ }^{1}$ id https://orcid.org/0000-0001-5511-2112 \\ Maria José López Montesinos ${ }^{1}$ id https:/orcid.org/0000-0001-6084-2670
}

\section{Como citar:}

Hellín Gil MF, Seva Llor AM, Roldán Valcárcel MD, Maciá Soler ML, Mikla M, López Montesinos MJ. Validação qualitativa de uma escala para medir a carga de trabalho de enfermagem em unidades de internação. Acta Paul Enferm. 2022;35·eAPE01961.

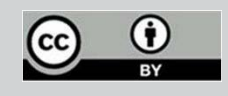

Descritores Hospitalização; Administração de recursos humanos: Serviço hospitalar de enfermagem; Processo de enfermagem; Recursos humanos de enfermagem no hospital

Keywords

Hospitalization; Nursing process: Nursing service, hospital; Nursing staff, hospital; Personnel management

\section{Descriptores}

Administración de personal; Hospitalización; Personal de enfermería en hospital; Proceso de enfermería; Servicio de enfermería en hospital

Submetido 24 de Julho de 2020

Aceito 23 de Março de 2021

\section{Autor correspondente}

Fuensanta Hellín G

Email: mfuensanta.hellin@carm.es

Editor Associado (Avaliação pelos pares): Camila Takao Lopes Escola Paulista de Enfermagem, Universidade Federal de São Paulo, São Paulo, SP, Brasil

\section{Resumo}

Objetivo: Projetar e validar uma escala para medir a carga de trabalho de enfermagem em Unidades de Internação de Adultos com base na Classificação de Intervenções de Enfermagem (NIC).

Métodos: Estudo analítico, descritivo, observacional, prospectivo, utilizando principalmente metodologia de pesquisa qualitativa, por meio de grupos focais, comitê de especialistas e entrevistas individuais, para validar uma Escala de Medição da Carga de Trabalho em Unidades de Internação de Adultos, a partir das intervenções selecionadas (NIC), com base nas características inerentes a tais unidades. Foi utilizada seleção aleatória para determinar os participantes (enfermeiros e especialistas) e é representativa das unidades de hospitalização em estudo. 0 estudo foi realizado de outubro de 2018 a abril de 2019.

Resultados: Não apenas os grupos focais, mas também as entrevistas individuais e o comitê de especialistas demonstraram concordância sobre a importância de se obter um instrumento para medir a carga de trabalho validado pelos próprios profissionais e representativo de seu trabalho, com linguagem padronizada e adaptada à saúde real. A análise quantitativa conduzida pelo comitê de especialistas mostra uma maior relevância $(91,67 \%)$ nos itens: "Prevenção" e "Educação para a saúde", bem como a consistência com a construto e a redação apropriada em 99\% dos itens selecionados. Os avaliadores mantiveram a ação em todos os itens com uma taxa de aceitação de $75 \%$ a $100 \%$.

Conclusão: 0 estudo qualitativo realizado fornece os dados necessários para a concepção e validação de uma escala para medir as cargas de trabalho de enfermagem, identificadas a partir das intervenções de enfermagem (NIC), como indicadores da gestão de recursos humanos.

\section{Abstract}

Objective: To design and validate a scale to measure nursing workloads in Adult Hospitalization Units based on Nursing Interventions (NIC).

Methods: Analytical, descriptive, observational, prospective study, using mainly qualitative research methodology, by means of focus groups, committee of experts and individual interviews, to validate a Workload Measurement Scale in Adult Hospitalization Units, from the selected interventions (NIC) on a basis of the inherent characteristics of such units. A random selection was used to determine the participants (nurses and experts) and it is representative of the hospitalization units under study. It was carried out from October 2018 to April 2019.

Results: Not only the focus groups but also the individual interviews and the committee of experts conducted agree on the importance of obtaining an instrument to measure workloads validated by the professionals themselves and representative of their work, with standardized language and adapted to actual healthcare.

'Murcian Institute of Biosanitary Research-Arrixaca, University of Murcia, Murcia, Spain.

University of Alicante, Alicante, Spain.

Conflitos de interesse: nada a declarar. 
The quantitative analysis conducted by the committee of experts shows a highest relevance $(91,67 \%)$ in the items: "Prevention" and "Health education", as well as consistency with the construct and appropriate wording in $99 \%$ of the items selected. Evaluators maintained the action on all items with a $75 \%$ to $100 \%$ acceptance rate.

Conclusion: The qualitative study carried out provides the necessary data for the design and validation of a scale to measure nursing workloads, identified from the nursing interventions (NIC), as indicators of human resources management.

\section{Resumen}

Objetivo: Proyectar y validar una escala para medir la carga de trabajo de enfermería en unidades de internación de adultos con base en la Clasificación de Intervenciones de Enfermería (NIC).

Métodos: Estudio analítico, descriptivo, observacional, prospectivo, que utilizó principalmente metodología de investigación cualitativa, por medio de grupos focales, comité de especialistas y entrevistas individuales, para validar una Escala de Medición de Carga de Trabajo en Unidades de Internación de Adultos, a partir de las intervenciones seleccionadas (NIC), con base en las características inherentes a tales unidades. Se utilizó selección aleatoria para definir los participantes (enfermeros y especialistas), que es representativa de las unidades de hospitalización en estudio. El estudio fue realizado de octubre de 2018 a abril de 2019.

Resultados: No solo los grupos focales, como también las entrevistas individuales y el comité de especialistas demostraron concordancia sobre la importancia de obtener un instrumento para medir la carga de trabajo, validado por los propios profesionales y representativo de su trabajo, con un lenguaje estandarizado y adaptado a la salud real. El análisis cuantitativo conducido por el comité de especialistas muestra una mayor relevancia (91,67 \%) en los ítems: "Prevención" y "Educación para la salud", así como una consistencia respecto a la elaboración y redacción apropiada en el 99 \% de los ítems seleccionados. Los evaluadores mantuvieron la acción en todos los ítems con un índice de aceptación de 75 \% a 100 \%.

Conclusión: El estudio cualitativo realizado proporciona los datos necesarios para la elaboración y validación de una escala para medir la carga de trabajo en enfermería, identificada a partir de las intervenciones de enfermería (NIC), como indicadores de la gestión de recursos humanos.

\section{Introdução}

Os recursos humanos em enfermagem devem ser gerenciados de acordo com um plano de necessidades, com o objetivo de planejamento, considerando os recursos econômicos e materiais existentes e os critérios de saúde que serão essenciais para determiná-lo, como pode ser visto atualmente na situação de pandemia da COVID-19 que vivemos. ${ }^{(1)}$

A análise e interpretação desses critérios causam as variaçóes que podemos ver em nosso mapa de saúde, ao relacionar as diferentes necessidades de recursos humanos, demonstrando que o que Domínguez Alcón disse em 1984 sobre gestão, estratégias e provisão de profissionais de saúde ainda é muito relevante hoje. ${ }^{(2)}$

Determinar o número adequado de pessoal de enfermagem de acordo com as necessidades dos pacientes, e a consequente carga de trabalho que essas necessidades geram, é essencial para fornecer cuidados de qualidade, segurança do paciente e equilíbrio na gestáo econômica de custo-benefício em qualquer sistema de saúde. ${ }^{(3)}$

$\mathrm{Na}$ Espanha, as escalas NEMS e NAS são os instrumentos mais utilizados para medir as cargas de trabalho, em Unidades de Cuidados Intensivos e de Ressuscitaçáo de Cuidados Críticos, e as Intervenções de Enfermagem não estão devidamen- te incluídas nestas escalas. Isto permitiria uma avaliação adequada das cargas de trabalho e do tempo investido em tais intervençóes, o que facilitaria a determinação da necessidade de recursos humanos. ${ }^{(4,5)}$

Em referência ao uso adequado desses recursos de enfermagem, tratamos de dois aspectos fundamentais, a qualificação dos profissionais e sua correta quantificação. ${ }^{(5)} \mathrm{A}$ "Qualificação", referente às competências profissionais que permitem a aplicação da assistência à saúde com base em uma metodologia e taxonomia com um quadro próprio de conhecimentos, e "Quantificaçáo", com respeito à discussão permanente sobre a demanda de profissionais e políticas de contenção de custos, resultando em fórmulas que se baseiam mais em fatores quantitativos do que qualitativos. ${ }^{(6,7)}$

Portanto, são necessários instrumentos para medir e quantificar a carga de trabalho, adaptados às necessidades e demandas reais do atendimento ao paciente e, por sua vez, expressos em uma terminologia padronizada semelhante à fornecida pelas intervençóes de enfermagem, de modo que a linguagem utilizada seja reconhecida internacionalmente e habitualmente aplicada por todos os profissionais de saúde. ${ }^{(8,9)}$

A mensuração da carga de trabalho é uma ferramenta útil para conhecer as tarefas do "cuidar", e o tempo necessário para cada uma delas. Estudos 
realizados sobre este assunto demonstram que as cargas de trabalho incluem a identificação, listagem de atividades e mensuração do tempo gasto, ${ }^{(9)}$ destacando as numerosas consequências negativas, quando há um desequilíbrio entre a carga de trabalho e a quantidade de recursos humanos disponíveis (erros na assistência médica, distúrbios psicossomáticos e baixa qualidade de vida do profissional enfermeiro, aumento da morbidade e mortalidade do cliente-paciente...). ${ }^{(4,7-14)}$

Em Enfermagem, a segurança do paciente está associada à incorporação de planos de cuidados individualizados para cada paciente, de acordo com a taxonomia de enfermagem da NANDA I(NANDA International), ${ }^{(15)}$ aceita internacionalmente. Tornase indispensável que o trabalho de enfermagem seja definido por meio da NIC (Nursing Interventions Classification), ${ }^{(16)}$ como recomendado em nosso país pelo Ministério da Saúde. ${ }^{(17)}$

As escalas de avaliação da carga de trabalho de enfermagem fornecem a classificação do paciente de acordo com suas necessidades de atendimento, promovendo o controle de qualidade e a comparação entre unidades com características similares, bem como objetivando a carga de trabalho e as necessidades específicas da equipe exigidas pela unidade ou serviço. Isto otimiza os recursos humanos de enfermagem e a gestão dos gastos com a saúde, como demonstram diversos estudos, a maioria focada em unidades especiais no ambiente hospitalar. ${ }^{(18-20)}$

Nossa pesquisa focou na necessidade de uma ferramenta que mensure a carga de trabalho de acordo com as intervençóes realizadas pelos enfermeiros nas unidades de hospitalização, com uma linguagem padronizada e adaptada ao atendimento de saúde real. O objetivo desta pesquisa foi desenvolver e validar uma escala para medir a carga de trabalho de enfermagem em Unidades de Hospitalização de Adultos, com base nas intervençóes NIC.

Este estudo corresponde à primeira fase de um projeto de pesquisa apoiado financeiramente pelo Instituto de Saúde Carlos III, em relação ao Plano Nacional Espanhol de Pesquisa Científica e Técnica e Inovação 2017/2020, destinado a projetos e iniciativas de pesquisa em serviços de saúde, como prioridade de pesquisa no desafio "Saúde, mudanças demográficas e bem-estar”, e inserido no "Programa Operacional Multirregional Espanhol FEDER (POPE) 2014-2020” (PI18/00950).

\section{Métodos}

Este projeto foi elaborado para garantir o intercambio de conceitos entre as diferentes açóes profissionais dos enfermeiros, tais como gerenciamento, ensino, pesquisa e atendimento ao paciente, no qual podemos incluir as intervençóes NIC. Estas refletem a carga de trabalho de enfermagem realizada nas unidades de hospitalização, de acordo com as exigências dos pacientes em situações reais.

A primeira fase da pesquisa consistiu na elaboração e validação de uma escala para mensurar a carga de trabalho de enfermagem para unidades de hospitalização de adultos. Os itens foram definidos de acordo com terminologia de enfermagem das intervençóes NIC, ${ }^{(13)}$ e uma primeira validação foi realizada por meio de metodologia qualitativa que consiste em: grupos focais, entrevistas individuais e um comitê de especialistas, até obtenção de uma escala que seria submetida a uma próxima fase de validação piloto, quantitativa e de estudo multicêntrico, a ser realizada posteriormente.

Desenho do estudo: Estudo analítico-descritivo, observacional e prospectivo, utilizando metodologia de pesquisa descritiva, qualitativa e quantitativa.

Metodologia: Foi realizada uma revisão documental descritiva, que consistiu de uma pesquisa bibliográfica sobre os principais instrumentos desenvolvidos para mensurar a carga de trabalho de enfermagem, baseada em intervençóes de enfermagem no campo hospitalar, como um indicador das necessidades de recursos humanos, publicada em espanhol e inglês nos últimos dez anos

A pesquisa foi realizada em bancos de dados científicos e repositórios digitais e sites credenciados utilizando operadores booleanos e palavras-chave dos descritores das ciências da saúde.

Após esta revisão bibliográfica, foi efetuada a análise da literatura. Assim, foi elaborado um esboço de escala para mensurar a carga de trabalho de enfermagem pela equipe de pesquisa, com a colabora- 
ção de um psicometrista - um especialista em escalas de medição. Esta escala incluiu as quatro funçóes da profissão de enfermagem: atendimento ao paciente, ensino, administração e pesquisa, cujos itens foram baseados em uma seleção de intervençóes NIC mais apropriadas para cada ação de enfermagem de acordo com as atividades e tarefas a serem realizadas nas diferentes Unidades de Internação.

Esta escala foi submetida a uma validação qualitativa, por meio de grupos focais, entrevistas individuais e um comitê de especialistas, reconhecidos nacional e internacionalmente nesta área. Também foi realizada uma análise descritiva quantitativa para complementar a qualitativa. A análise psicométrica concentrou-se em garantir a reprodutibilidade e validade da escala, descrevendo a confiabilidade no teste-reteste e interobservadores, a consistência interna e o poder discriminatório, com respeito à reprodutibilidade, bem como a validade de critério, aparência e conteúdo.

\section{Descrição detalhada das seguintes etapas e dos procedimentos metodológicos e análises realizadas, amostra e escopo do estudo:}

- Grupo focal: Técnica qualitativa, destinada a recolher as opinióes, contribuiçóes e propostas dos profissionais sobre a escala a ser validada. Foram constituídos dois grupos focais. O primeiro grupo com oito enfermeiros de unidades de hospitalização e, um segundo grupo com oito enfermeiros que não só realizam suas tarefas de saúde nestas unidades em um hospital de nível terciário, mas também são referências de qualidade. Foram realizadas entrevistas estruturadas e abertas nos dois grupos representativos dos enfermeiros, fornecendo suas experiências profissionais e pessoais e conhecimento sobre o tema da pesquisa para melhorar o desenho inicial da escala desenvolvida pela equipe de pesquisa. Cada entrevista durou aproximadamente duas horas e foi gravada em áudio e vídeo. Os dados foram analisados utilizando um software específico para metodologia qualitativa, Atlas.ti8.

- Entrevistas individuais: Dez entrevistas individuais semiestruturadas foram realizadas com enfermeiros e supervisores de unidades de hos- pitalização. Estas foram gravadas em áudio para posterior análise utilizando o programa Atlas.ti8. Os participantes de cada parte desta validação passaram por uma seleção aleatória. O princípio da saturação foi aplicado para determinar o número de entrevistas e o número de grupos focais necessários de tal forma que a amostra fosse representativa da maioria dos tipos de unidades de hospitalização (em termos de pacientes e especialidade médica).

- Comitê de Especialistas. Foi realizada uma dupla análise das contribuiçóes dos especialistas reconhecidos nacional e internacionalmente em cada item da escala:

- A análise quantitativa concentrou-se na avaliação da validade do conteúdo de cada item, e de toda a escala através das 12 sugestôes desses especialistas. Cada especialista avaliou individualmente cada item da escala, aplicando diferentes tipos de avaliação. Os dois primeiros analisaram itens relacionados ao construto, um avaliou os itens correspondentes à relevância que dão ao item para sua exploraçáo, por meio de uma escala Likert que vai de um a quatro sendo: $1=$ não relevante, $2=$ substancialmente relevante; $3=$ muito relevante e $4=$ =levada relevância, e o outro especialista analisou sua consistência, avaliando-a como "consistente" ou "não consistente".

Além disso, expressaram sua opinião sobre a redação e compreensão do item, avaliando-o como: "pobre", "aceitável” ou "bom", e qual seria sua ação a seguir com cada um deles, de acordo com as avaliaçóes anteriores: "manter", "mudar" ou "remover". Esta análise foi realizada utilizando o SPSS Statitics program.

- Para a análise qualitativa, foi incluída uma seçáo no final de cada bloco. Um espaço, a ser preenchido individualmente, para "comentários" sobre a escala em geral e sobre cada item, e também um local onde cada decisão deveria ser esclarecida, em casos de avaliação negativa para qualquer um dos aspectos em avaliação. Havia também um 
espaço para "sugestôes", na qual eles poderiam expor suas propostas relacionadas a avaliação geral da escala e avaliaçáo individual de cada item, expressando se consideravam apropriado incorporar novos itens, ou funçóes de enfermagem que ainda estavam por serem exploradas ou incompletas. Para o comitê de especialistas, foi elaborado um modelo da escala inicial, incorporando as modificaçóes propostas pelos grupos focais e entrevistas individuais e, incluindo as escalas de Likert com a pontuação codificada explicada acima. Estas informaçôes foram enviadas por e-mail, juntamente com uma carta-convite agradecendo sua participação no comitê. Elas foram registradas no Escritório de Propriedade Intelectual (Intellectual Property Office).

- Linha do tempo: Esta fase do projeto foi realizada nos últimos três meses de 2018, para o projeto inicial e grupos focal, e durante os primeiros quatro meses de 2019 para as entrevistas individuais e o comitê de especialistas.

- Aspectos éticos: As permissóes correspondentes foram solicitadas ao Comitê de Ética, mantendo a confidencialidade, conforme estabelecido na Lei 15/1999, de 13 de dezembro, relativa a Proteção de Dados Pessoais (PDCP, siglas em espanhol), e o Regulamento Geral sobre a Proteção de Dados (RGPD) (UE) 2016/679, de 27 de abril.

\section{Resultados}

Após realização dos grupos de discussão e entrevistas individuais, pudemos observar concordância entre as declaraçóes, opinióes e sugestóes dos participantes. Após analisados com o Altlas.ti8, estes dados foram esquematizados por meio de uma rede de códigos atribuídos a cada tópico comum discutido nas diferentes metodologias, e ao qual associamos uma citação textual proposta pelos participantes, representativa daquele código, e que identificava a opinião comum, após identificar a saturação dos resultados.
Sua inter-relação pode ser observada, demonstrando como os participantes valorizaram a escala, seus itens, as funçóes dos profissionais envolvidas, sua visão da profissão, gestão de recursos humanos e a importância de mensurar a carga de trabalho para melhorar a qualidade dos cuidados prestados pelos enfermeiros aos seus pacientes (Figura 1).

Com base na escala para medir cargas de trabalho, algumas questóes importantes para a enfermagem, como a gestão, se destacam, demonstrando a importância de ser esta uma competência de enfermagem, onde a medida de cargas de trabalho desempenha um papel fundamental, bem como sua relação com as possíveis repercussóes que poderia ter nos pacientes e profissionais (Figura 1).

Também, a relação da escala em estudo com a importância da participação dos profissionais de saúde, tanto em seu desenho quanto em sua validação, a fim de facilitar e melhorar sua aplicabilidade futura, par que possa ser globalmente representativa de todo o trabalho realizado pelos enfermeiros nas unidades de hospitalização de adultos, considerando os aspectos de elaboracao da escala, tais como a escolha dos itens mais apropriados com base nas intervençóes NICs, e o tempo atribuído para realizá-los, bem como as diferentes açóes de enfermagem incluídas (Figura 1).

Todas estas avaliaçóes estão relacionadas à importância deste estudo, para que os profissionais de saúde sejam inovadores e para a escala proposta pelo grupo de pesquisa, uma vez que não foram encontrados estudos ou pesquisas que mostrem escalas para medir cargas de trabalho, associadas a intervenções NIC, para unidades de hospitalização, como apresentado neste artigo.

Com relação aos resultados obtidos com o comitê de especialistas, na análise descritiva quantitativa, podemos notar que os itens mais valorizados em termos de relevância e, referidos com "relevância alta” (91,67\%) foram Prevenção e Educação em Saúde e Coleta e Manejo de amostras com menor relevância (Figura 2).

Os seguintes itens obtiveram uma taxa de $100 \%$ de consistência com o constructo: Ajuda para o autocuidado, Prevenção, Medicação, Educação em saúde, Gestão da nutrição, Cuidados com dispositivos, Mudança de 


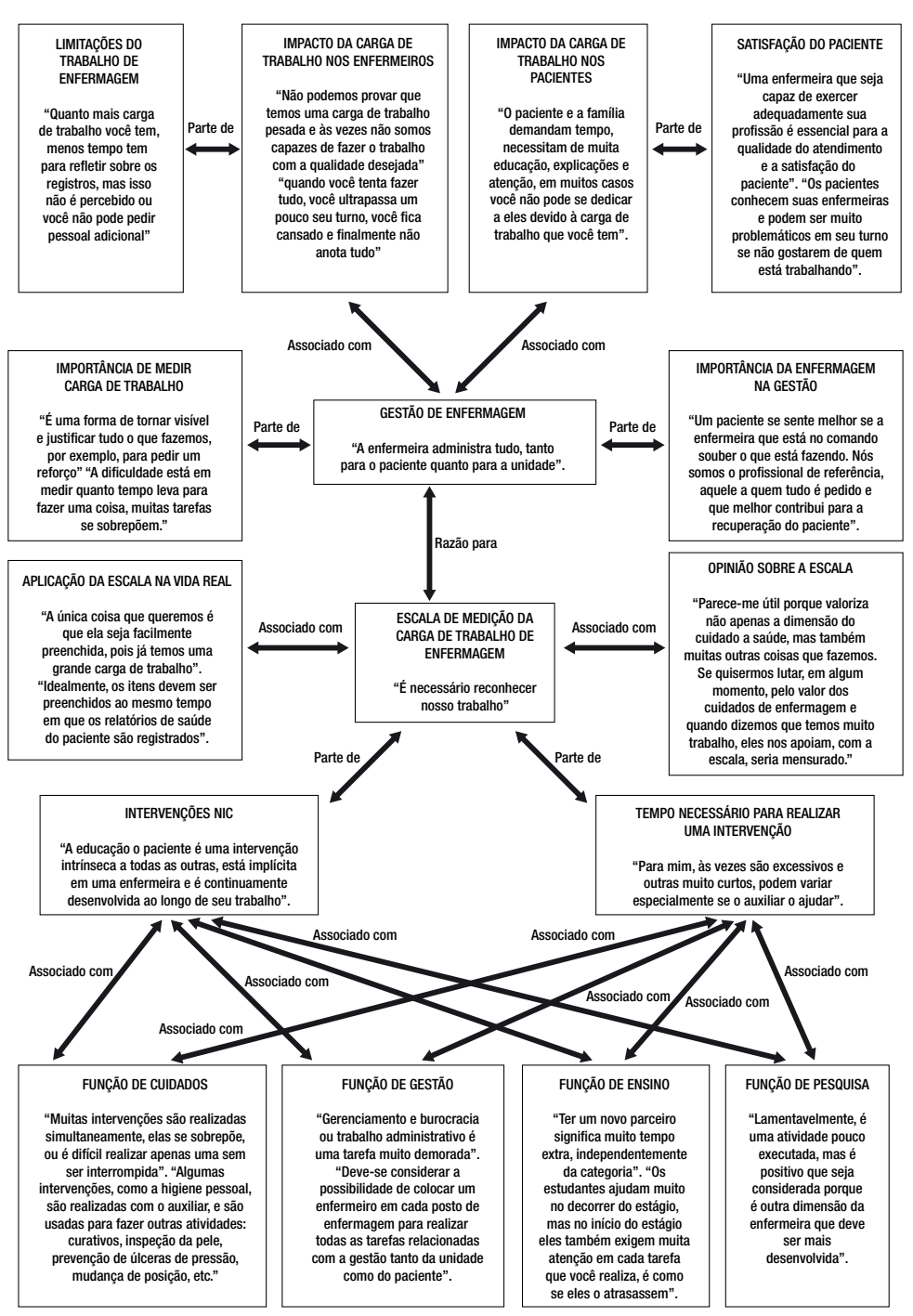

Figura 1. Análise de grupos focal e entrevistas individuais com enfermeiros

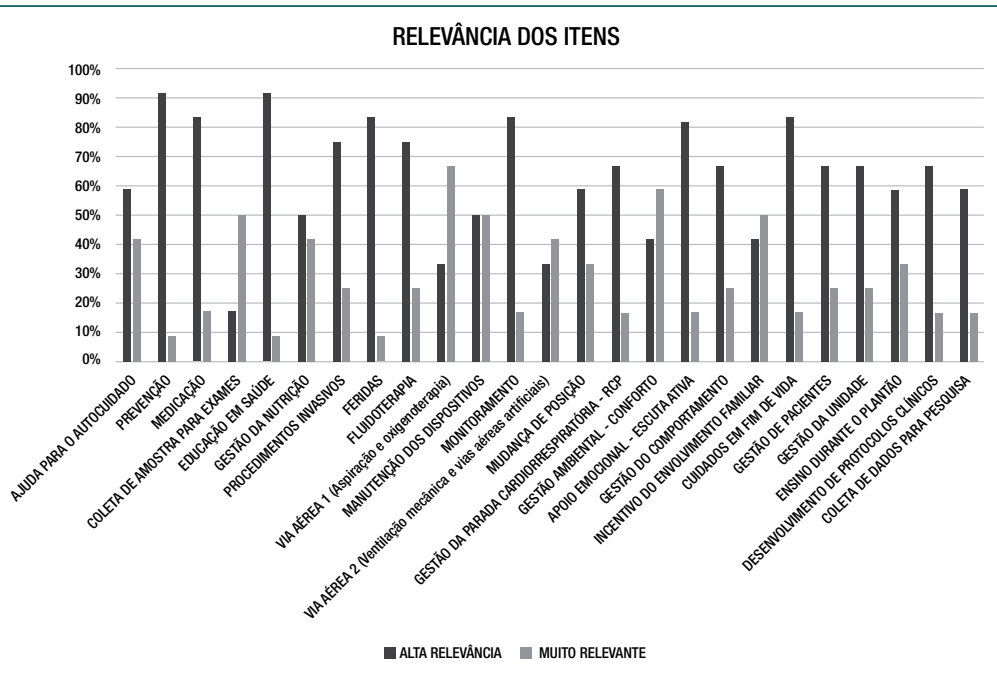

Figura 2. Relevância dos itens referidos pelos especialistas como "relevância alta "e" muito relevante" 


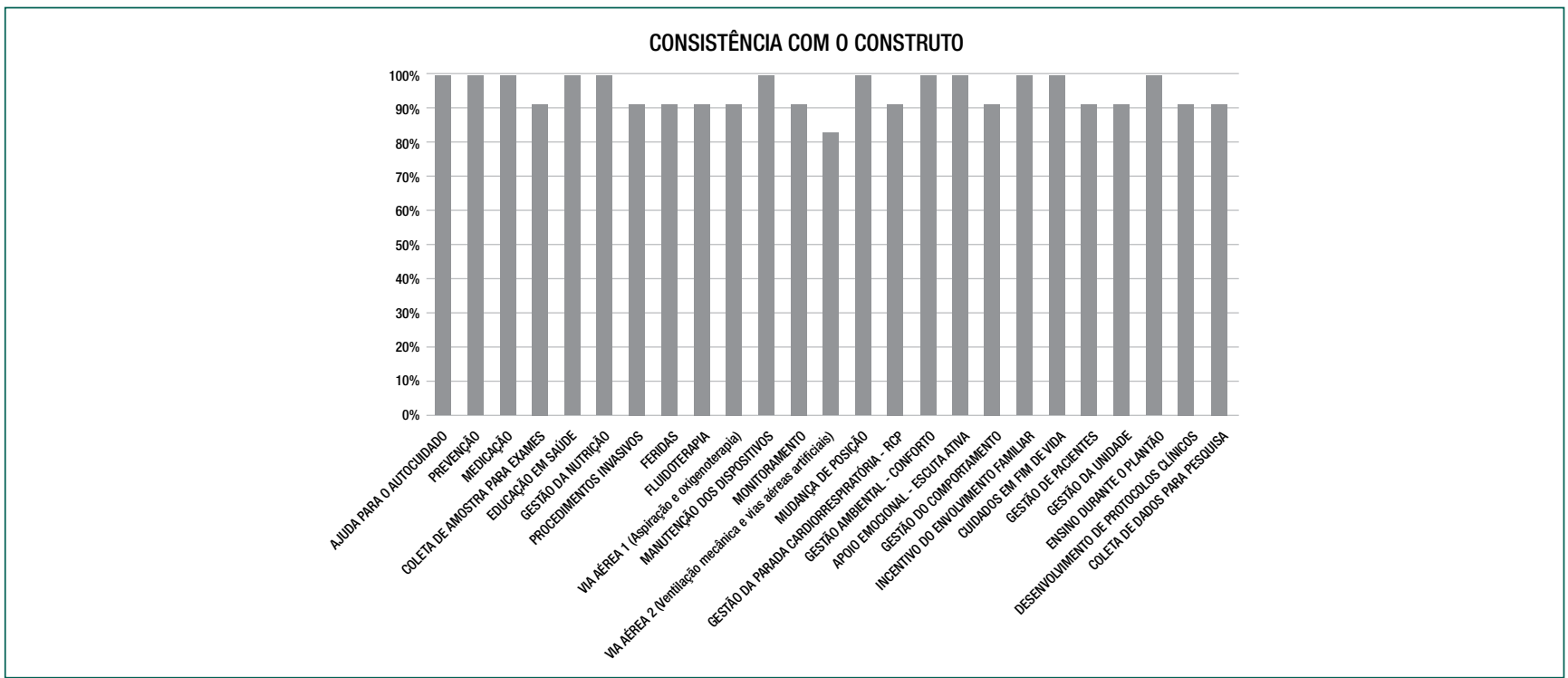

Figura 3. Consistência dos itens de acordo com o comitê de especialistas

posição, Gestão ambiental: conforto, Apoio emocional-escuta ativa, Incentivo ao envolvimento familiar, Cuidados no final da vida e Ensino durante o turno de trabalho. Todos os itens com consistência mínima de 83,33\% foram avaliados (Figura 3).

Com relação à redação, os itens mais valorizados foram: Educação em saúde com uma taxa de avaliação de $83,33 \%$, considerada aceitável, seguido por Mudança de posição com $75 \%$.

$\mathrm{O}$ avaliador decidiu manter o item proposto com uma taxa de 100\%: Ajuda para o autocuidado, Prevenção, Monitoramento, Mudança de posição e Cuidados em fim de vida.

Quanto à análise qualitativa obtida a partir das contribuiçóes e comentários dos especialistas consultados, estas coincidem com as declaraçóes emitidas tanto nos grupos de discussão como nas entrevistas em profundidade, destacando as coincidências nos seguintes tópicos:

- A dificuldade em compreender alguns itens devido à falta de familiaridade com a terminologia NIC.

- A controvérsia sobre os tempos atribuídos a cada um dos itens a serem realizados, (tempos padronizados pela NANDA I), que na maioria das vezes náo estáo de acordo com o cuidado real do paciente, seja em excesso ou por padrão.

- A importância de obter uma ferramenta de medição da carga de trabalho para unidades de hospitalização que facilite a gestão de recursos humanos de enfermagem de acordo com as reais demandas de cuidado ao paciente.

- O sucesso de se ter uma ferramenta de medição que inclua todas as intervençóes realizadas pelos enfermeiros, desde a assistência à saúde até a função de gerenciamento, incluindo o ensino que é realizado continuamente com pacientes e colegas, assim como a pesquisa.

\section{Discussão}

A medida da carga de trabalho de enfermagem requer ferramentas adaptadas às características das unidades a serem avaliadas. Por esta razão, se tivermos que medir a carga de trabalho de enfermagem em unidades de internação, não podemos utilizar outras ferramentas existentes criadas para outros tipos de unidades, como as utilizadas para Cuidados Intensivos, ${ }^{(4,10,18)}$ mas devem ser instrumentos adaptados à própria atividade de enfermagem realizada na internação, incluindo todas as funçôes da profissão de enfermagem.

Esta reflexão também aparece em outros estudos, ${ }^{(21)}$ nos quais se destaca o potencial das ferramentas de medição da carga de trabalho para ajudar na tomada de decisóes relacionadas à equipe de enfermagem, mas uma abordagem mais holística é necessária para 
considerar fatores adicionais para garantir que modelos sólidos de planejamento sejam desenvolvidos.

Neste processo de adaptação, é essencial utilizar a linguagem padronizada de enfermagem proposta pela NANDA I ${ }^{(15)}$ e dentro deste sistema, as intervençóes $\mathrm{NIC},{ }^{(16)}$ como um modelo para o desenvolvimento de medidas para a carga de trabalho de enfermagem. ${ }^{(22)}$

Como em outros estudos, ${ }^{(9)} \mathrm{o}$ uso das intervençôes $\mathrm{NIC}^{(16)}$ também foram adotadas como referência para medir cargas de trabalho em equipes de enfermagem, ${ }^{(9,13,22-24)}$ mas não foram desenvolvidas de forma global e válida para qualquer unidade de hospitalização de adultos, mas focadas em unidades específicas classificadas por especialidades médicas. ${ }^{(21,23,24)}$

Também encontramos estudos recentes que utilizaram metodologia qualitativa para validar ferramentas, de maneira semelhante ao estudo que apresentamos e apresentam uma análise crítica para melhorar a ferramenta inicialmente proposta. ${ }^{(9,23)}$ Entretanto, enfocam algumas especialidades médicas específicas, que definem um tipo de paciente com um perfil particular, que requer cuidados muito específicos tais como, unidades oncológicas ${ }^{(9,23)}$ ou unidades de saúde mental, ${ }^{(21)}$ no lugar de um instrumento que pode ser usado em qualquer unidade de hospitalização de adultos. Um instrumento que considere todas as funçóes de enfermagem, não apenas o atendimento ao paciente, embora este seja o mais comumente associado à profissão de enfermagem, mas também a função gerencial, como ensino e pesquisa, cada vez mais presentes na profissão.

Em nosso estudo, assim como em outros consultados, ${ }^{(9,22,23,25)}$ é importante equilibrar o conhecimento das intervençôes $\mathrm{NIC}^{(16)}$ e a experiência prática dos cuidados das unidades que se deseja avaliar. É por isso que decidimos dar voz aos profissionais, por meio de diferentes metodologias, para conferir sua concordância de forma qualitativa, enriquecer o instrumento que resulta desta validação, refletir a real atenção à saúde deste tipo de unidade e, consequentemente, facilitar sua aplicabilidade e assimilação por profissionais que a necessitam, de acordo com suas próprias opinióes encontradas em outras publicaçóes, ${ }^{(20)}$ para melhorar a gestão de recursos humanos e melhorar a qualidade da atenção que prestam aos seus pacientes.

\section{Conclusão}

A realização deste estudo com metodologia qualitativa, como primeira fase de um projeto, constitui o início fundamental para projetar e validar uma escala destinada a medir a carga de trabalho dos enfermeiros que trabalham em unidades de internação. A adoção de uma escala validada (objetivo global do projeto) para medir a carga de trabalho em unidades de internação, associada às Intervençóes NIC, dimensionada e validada a partir das opiniôes, experiências e contribuiçóes dos profissionais, por meio dos instrumentos de metodologia qualitativa utilizados, objetivo deste estudo, evidencia uma adaptação à situação real em que será utilizada, já que os próprios profissionais redesenham e validem a escala que poderiam utilizar. Este estudo confirma a necessidade existente nos profissionais de saúde em medir e quantificar a carga de trabalho que eles suportam associada às intervençóes de enfermagem propriamente ditas realizadas em todas as unidades de hospitalização. Estas intervenções são sempre o indicador mais confiável para determinar a quantidade necessária de pessoal de enfermagem e esta escala uma ferramenta de gestão relevante por combinar os recursos humanos de enfermagem com as demandas e necessidades reais do paciente, visando melhorar a qualidade do atendimento, a segurança do paciente enquanto aperfeiçoam a atividade de enfermagem profissional. A originalidade e a importância desta pesquisa residem no fato de que foi realizada em unidades de internação hospitalares, serviços que, apesar de utilizarem terminologia de enfermagem, não aplicam ferramentas de mensuração da carga de trabalho relacionadas às intervençóes reais de enfermagem utilizadas nestes serviços, já que tradicionalmente são mais desenvolvidas em unidades de terapia intensiva.

\section{Agradecimentos}

Nossos sinceros agradecimentos a todos os profissionais de enfermagem que participaram deste estudo, tanto nos grupos focal como nas entrevistas individuais e no comitê de especialistas. Sem eles nunca teríamos realizado o estudo. Agradecemos 
por todas as contribuiçóes, opinióes e sugestóes, que serão o elemento-chave para o desenvolvimento do nosso projeto de pesquisa no qual o estudo aqui apresentado está incluído. Também gostaríamos de agradecer ao Instituto de Saúde Carlos III, por ter selecionado nosso projeto em sua convocação - AES 2018, e por seu apoio financeiro dentro do "Programa Operativo FEDER Plurirregional de España (POPE) 2014-2020” (PI 18/00950).

\section{Colaborações}

\section{Hellín Gil MF, Seva Llor AM, Roldán Valcárcel MD, Maciá Soler ML, Mikla M e López Montesinos MJ, contribuíram para a elaboração do projeto, análise e interpretação de dados, redação de artigos, revisão crítica relevante do conteúdo intelectual e aprova- ção da versão final a ser publicada.}

\section{Referências}

1. Martinez Estalella G, Zabalegui A, Sevilla Guerra S; en nombre del Comité Técnico de la Dirección Enfermera (CTDE). Gestión y liderazgo de los servicios de Enfermería en el plan de emergencia de la pandemia COVID-19: la experiencia del Hospital Clínic de Barcelona. Enferm Clin. 2021;31:S12-7.

2. Dominguez Alcon C. Cuadernos de La Enfermera. Administración, legislación. Barcelona: Masson; 1984.

3. Soares MI, Resck ZM, Camelo SH, Terra FS. Gerenciamento de recursos humanos e sua interface na sistematização da assistência de enfermagem. Rev Enfermería Global. 2016;42:341-52.

4. Hellín Gil MF. Adecuación de un instrumento de medida de cargas de trabajo y análisis de su nivel de cumplimiento en una Unidad de Cuidados Intensivos: propuesta de oportunidad de mejora [tese]. Murcia: Universidad de Murcia; 2015.

5. Miranda DR. El impacto de la organización sobre la calidad de la medicina intensiva. Presentación de un estudio europeo. EURICUS-I. Rev Calid Asist. 1996;11:S74-9.

6. Abad-Corpa E, Molina-Durán F, Vivo-Molina MC. Estudio RN4CAST en Murcia: características organizacionales de los hospitales y perfiles enfermeros. Rev Calid Asis. 2013;28(6):345-54.

7. Aiken LH, Cerón C, Simonetti M, Lake ET, Galiano A, Garbarini A, et al. Hospital nurse staffing and patient. Rev Méd Clín Condes. 2018;29(3):322-7.

8. Berry L, Curry P. Nursing workload and patient care: understanding the value ofnurses, the effects of excessive workload, and how nursepatient ratios and dynamic staffing models can help. Ottawa: Canadian Federation of Nurses Unions; 2012 [Cited 2020 May 15]. Available from: https://nursesunions.ca/wp-content/uploads/2017/07/ cfnu workload_printed_version_pdf.pdf
9. Souza CA, Jericó MC, Perroca MG. Nursing intervention/activity mapping at a Chemotherapy Center: an instrument for workload assessment. Rev Lat Am Enfermagem. 2013;21(2):492-9.

10. Pérez Niculcar PN, Zuazua Rico D. Carga de trabajo de enfermería en la unidad de cuidados intensivos de adultos. Revisión sistemática. Rev Nure Inv. 2018;15(93):2-17. Review.

11. López Montesinos MJ. Psychosocial study about the consequences of work in hospital nurses as human resource management. Rev Lat Am Enfermagem. 2013;21(Spec):61-70

12. Puerto Cremades J, Maciá Soler L, López Montesinos MJ, Pedraz Marcos A, González Chorda VM. Uma nova contribuição para a classificação dos fatores estressores que afetam os profissionais de enfermagem. Rev Lat Am Enfermagem. 2017;25:e2895.

13. Possari JF, Gaidzinski RR, Lima AF, Fugulin FM, Herdman TH. Use of the nursing intervention classification for identifying the workload of a nursing team in a surgical center. Rev Lat Am Enfermagem. 2015;23(5):781-8.

14. Somensi RM, Aquino Caregnato RC, Cervi GH, Dias Flores C. Workload: a comparison between the online and observational methods. Rev Bras Enferm. 2018;71(4):1850-7.

15. Carpenito LJ. Manual de diagnósticos enfermeiros. 15a ed. Filadélfia: Lippincott Williams \& Wilkins; 2017.

16. Bulechek B, Bulechek G, Dochterman W, Wagner C. Clasificación de Intervenciones de Enfermería (NIC). 7a ed. España: Elsevier; 2018.

17. Madrid. Ministerio de Sanidad. Servicios Sociales e Igualdad. Estrategia de Seguridad del Paciente del Sistema Nacional de Salud: período 2015-2020. Madrid: Ministerio de Sanidad; 2016.

18. Santos Nobre RA, Nascimento Rocha HM, Jesus Santos F, Dantas dos Santos A, Gois de Mendonça R, Freire de Menezes A. Aplicación del Nursing Activities Score (NAS) en diferentes tipos de UCl's: una revisión integrativa. Enferm Glob. 2019;18(56):485-528.

19. Hellín Gil MF, López Montesinos MJ, Seva Llor AM, Ferrer Bas MP, Maciá Soler ML. Adecuación de escalas para medir cargas de trabajo mediante metodología de calidad. Rev Bras Enferm. 2017;70(1):3946.

20. Hellín Gil MF, López Montesinos MJ, Seva Llor AM, Ferrer Bas MP, Maciá Soler ML. Valoración de dos escalas de medición de cargas de trabajo por profesionales de enfermería. Rev Enferm Atenção Saúde. 2017;6(1):18-29.

21. Fanneran T, Brimblecombe N, Bradley E, Gregory S. Using workload measurement tools in diverse care contexts: the experience of staff in mental health and learning disability inpatient settings. J Psych Mental Helth Nurs. 2015;22(10):764-72.

22. De Cordova PB, Lucero RJ, Hyun S, Quinlan P, Price K, Stone PW. Usar la clasificación de intervenciones de enfermería como una medida potencial de la carga de trabajo de la enfermera. J Cuid Enferm. 2010;25(1):39-45

23. Martin LG, Gaidzinski RR. Creating and validating an instrument to identify the workload at an Oncology and Hematology Outpatient Service. einstein (São Paulo). 2014;12(3):323-9.

24. Campos MS, Oliveira BA, Perroca MG. Workload of nurses: observational study of indirect care activities/interventions. Rev Bras Enferm. 2018;71(2):297-305.

25. Cruz CW, Gaidzinski RR. Nursing time in a diagnostic imaging center: development of an instrument. Acta Paul Enferm. 2013;26(1):7985. 